\title{
Prophylactic Gastric Histopathological Evaluation of Fluvoxamine and Mirtazapine in Aspirin Induced Rat Model
}

${ }^{1}$ Shahnaz Fatima, ${ }^{2}$ Maira Bhatti, ${ }^{3}$ Saadia Shahzad Alam

${ }_{1}$ Department of Pharmacology Sahara Medical College, Narowal

${ }^{2}$ Department of Pharmacology Sharif Medical College, Lahore

${ }^{3}$ Department of Pharmacology, Shaikh Zayed Medical Complex, Lahore

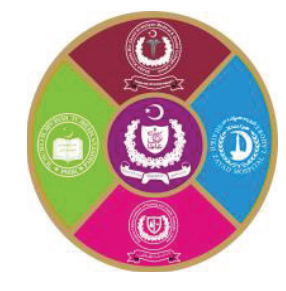

\begin{abstract}
Introduction: Depression is a mood disorder characterized by emotional dysregulation that causes distress in patients. It has strong association with peptic ulcer due to the underlying factor, stress, involved in its pathogenesis. The increased usage of NSAIDS and associated peptic ulcer disease (PUD) in depressive patients has raised concerns. Standard treatment is with PPIs like omeprazole Antiulcer effect of antidepressants fluvoxamine and mirtazapine was investigated in our previous research work by comparing the gastric glutathione and PGE2 levels in aspirin induced ulcer in rats. Aims \& Objectives: The current research aimed to comparatively evaluate the histopathological changes caused by fluvoxamine, mirtazapine vs omeprazole in gastric antral tissue by using aspirin. Place and duration of study: A cross sectional analytical research of one month duration was conducted in Federal Postgraduate Medical Institute, Shaikh Zayed Medical Complex, Lahore. Material \& Methods: Five groups of Albino rats with each group comprising of ten animals, were used. Group A received aspirin only as a single dose of $400 \mathrm{mg} / \mathrm{kg}$ via nasogastric tube. Groups B, C, D and E received through oral route prophylactically and in single dose omeprazole $20 \mathrm{mg} / \mathrm{kg}$, fluvoxamine $100 \mathrm{mg} / \mathrm{kg}$, fluvoxamine $200 \mathrm{mg} / \mathrm{kg}$ and mirtazapine $60 \mathrm{mg} / \mathrm{kg}$ respectively. Ulceration was induced with aspirin in groups B to $\mathrm{E}$ after forty five minutes. Four hours after administration of aspirin all the rat groups were killed by thiopentol $50 \mathrm{mg} / \mathrm{kg}$ intraperitoneally. Antral ulcerated tissue was taken and kept in 10\% formalin. Comparison was made for ulcers, necrosis, cellular infiltration, congestion. Results: Upon sacrificing a histopathological analysis of gastric mucosa revealed that in group A (aspirin treated only) all animals had ulceration with necrosis, $70 \%$ were of severe nature while $80 \%$ had severe cellular infiltration and congestion. In comparison mild ulceration was noted in $70 \%$ of animals in Group C (fluvoxamine $100 \mathrm{mg} / \mathrm{kg}$ ), $20 \%$ in group E (mirtazapine $60 \mathrm{mg} / \mathrm{kg}$ ) and none in group B (omeprazole $20 \mathrm{mg} / \mathrm{kg}$ ) and D (fluvoxamine $200 \mathrm{mg} / \mathrm{kg}$ ) and no necrosis. However all animals in groups B, C, D and E had mild cellular infiltration and congestion. Conclusion: Rat gastric histological analysis revealed significant prophylactic gastroprotective ability of $200 \mathrm{mg} / \mathrm{kg}$ fluvoxamine and lesser in $100 \mathrm{mg} / \mathrm{kg}$ fluvoxamine and $60 \mathrm{mg} / \mathrm{kgmirtazapine}$ versus $20 \mathrm{mg} / \mathrm{kg}$ omeprazole in aspirin induced damage.
\end{abstract}

Key words: Aspirin induced peptic ulcer, Proton pump inhibitors (PPIs), fluvoxamine, mirtazapine, omeprazole,

\section{INTRODUCTION}

$\mathrm{D}$ epression is type of mood disorder often unrecognized and untreated, that causes persistent feeling of sadness and loss of interest in routine activities. ${ }^{1}$ Stress not only plays a major role in pathogenesis of depression but is also linked to disturbance in hypothalamic pituitary axis. Thus both stress and depression elevate the risk of developing comorbidities like PUD. ${ }^{2}$

Gastroduodenal ulcer is a common type of peptic ulcer affecting $10 \%$ of global population. ${ }^{3,5}$ Peptic ulcers are excavations that are formed in the mucosal wall of the stomach, in the pylorus, in the duodenum or esophagus. ${ }^{4}$

Increased usage of NSAIDS especially aspirin by depressive patients have been strongly implicated in pathogenesis of gastric ulcer. The imbalance between protective factors such as secretion of mucous, bicarbonate, prostaglandins and offensive factors such as increased pepsin and acid secretion results in peptic ulceration. ${ }^{6}$ Another mechanism of gastric epithelial dysfunction is through downregulation of Claudine and members of Claudine protein family which plays important role in 
formation of tight junctions. ${ }^{7}$ Inhibition of prostaglandin is considered as most important in pathogenesis of NSAID's induced gastric damage. ${ }^{8}$ Aspirin also decreases the gastric mucosal glutathione. This reduction is due to increased formation of free radicals which eventually lead to peptic ulcer formation by diminishing blood flow, hindering restitution and impeding regeneration. ${ }^{9}$

Gastric ulcers are managed nowadays by multiple drugs. ${ }^{10}$ Latest studies showed that prevention and healing of ulcers caused by NSAIDS can be achieved by PPI's such as omeprazole. ${ }^{11}$ Apart from standard drugs for management of PUD, recently antidepressants like fluvoxamine, mirtazapine, venlafaxine and meclobomide have also begun to be researched for their antiulcer potential.

Gastroprotective effect of fluvoxamine and mirtazapine was determined through gastric GSH and PGE2 levels in our previous reasearch ${ }^{12}$. This study aims to further investigate the prophylactic effect of fluvoxamine, mirtazapine and omeprazole on histopathology of aspirin induced gastric ulcer.

\section{MATERIAL AND METHODS}

A total of 50 Albino rats weighing 190-210g were used for the study. They were fed a certified diet under an environmentally controlled condition.

Aspirin was selected for ulcer formation in rats due to fast induction time, $35 \%$ greater ability to produce ulcers than indomethacin ${ }^{19}$ and wide usage as NSAID in humans.

Followings materials were used for this study.

a) Cap omeprazole $20 \mathrm{mg}$, tablet fluvoxamine $50 \mathrm{mg}$ and tablet mirtazapine $30 \mathrm{mg}$ were procured from Fazal Din Pharmacy.

b) $10 \%$ formalin was used (Merck Germany).

It was a cross sectional analytical study. Animals were placed in Postgraduate Medical Institute Lahore. Histopathology was done at University of Veterinary and Animal sciences Lahore. All the rats were fasted for 24 hours.

All drugs were administered via orogastric tube in single dose.

Group A: Aspirin 400mg/kg

The drug administration in Group B, C, D \& E was followed 45 minutes later by Aspirin $400 \mathrm{mg} / \mathrm{kg}^{13,14}$

Group B: Omeprazole 20mg/kg

Group C: Fluvoxamine 100mg $/ \mathrm{kg}$

Group D: Fluvoxamine 200mg/kg

Group E: Mirtazapine $60 \mathrm{mg} / \mathrm{kg}$

Four hours after the administration of aspirin all the rat groups were sacrificed by giving Thiopentol injection at a dose of $50 \mathrm{mg} / \mathrm{kg}$ intraperitoneally. ${ }^{15}$ Abdomen was opened by giving a midline incision, stomach was excised and mucosa scrapings from ulcerated antrum were taken for histopathological evaluation of gastric mucosa and kept in 10\% formalin.

Collection of gastric antral tissue and preparation of Histopathological slides:

Vertical sections were obtained, processed and embedded in paraffin to form tissue blocks. Multiple slides with thickness raging 3-5 microns were made with hematoxylin and eosin for microscopic analysis.

\section{Histopathological criteria for ulceration:}

On microscopic examination criteria for aspirin induced ulceration was set as follows.

Grade 0: no ulceration

Grade 1: mild superficial erosion

Grade 2 :ulceration involving $2 / 3$ of mucosa moderate Grade 3: ulceration involving entire mucosa severe Inflammatory changes, congestion and necrosis were graded as mild, moderate and severe. Slides were examined at University of Veterinary and Animal Sciences at Histopathology Department. Photographs were taken and saved in computer.

\section{Statistical analysis:}

Data entry and its analysis was done by using SPSS 19.0. Cellular infiltration, congestion, ulceration and necrosis were compared by using chi square Test.

\section{RESULTS}

When animals were sacrificed and comparison was made for cellular infiltration it was noted that in group A (Aspirin pretreated) all 10 had cellular infiltration, 2 moderate and 8 of severe nature. The group B (Omeprazole $20 \mathrm{mg} / \mathrm{kg}$ ) had cellular infiltration in only 1 case of mild nature, group $\mathrm{C}$ (Fluvoxamine100mg $/ \mathrm{kg}$ ) had mild infiltration in all 10 animals, Group D (Fluvoxamine $200 \mathrm{mg} / \mathrm{kg}$ ) mild in 2 and Group E (Mirtazapine $60 \mathrm{mg} / \mathrm{kg}$ ) also has all 10 with mild infiltration. The pair wise difference among groups was significant for all pairs with $\mathrm{p}$ value $<0.001$ or 0.001 except the difference between group $\mathrm{C}$ and $\mathrm{E}$, where no difference was observable and between $\mathrm{B}$ and $\mathrm{E}$ where the difference was insignificant with the $p$ value1.000. When comparison was made for congestion it was noted in group A, all 10 had congestion, 2 moderate and 8 of severe nature, group $\mathrm{C}$ had mild congestion in 7 of the animals, Group D mild in 3 and group E had 2 with mild congestion. The difference was significant among groups with $\mathrm{p}$ value $<0.001$ when compared to Group A. Group C had significantly higher congestion as compare to group $\mathrm{B}$ with $\mathrm{p}$ value 0.002. Group $\mathrm{B}$ had no significant difference as 
compared to group $\mathrm{D}$ and $\mathrm{E}$ with $\mathrm{p}$ values 0.576 and 1.000 respectively. Similarly group D and E were different from $\mathrm{C}$ with $\mathrm{p}$ values 0.180 and 0.072.There was no significant difference between D and $\mathrm{E}$ with $\mathrm{p}$ value 1.000. When comparison was made for ulcers it was noted that in group A All 10 had ulcers, 3 moderate and 7 of severe nature. The group $\mathrm{B}$ and $\mathrm{D}$ had no ulcers in any of the animals. The group $\mathrm{C}$ had mild in ulcers in 7 of the animals and group $\mathrm{E}$ had 2 with mild ulcers. The difference was significant among groups with $p$ value $<0.001$. The pairwise difference among groups was significant for all 4 pairs wit $\mathrm{p}$ value $<0.001$ when compared to group A. Group $\mathrm{C}$ had significantly higher ulcers as compared to group B with $\mathrm{p}$ value 0.005. Group $B$ had no significant difference as compared to $\mathrm{D}$ and $\mathrm{E}$ with $\mathrm{P}$ values 0.005 and 0.465 respectively. similarly group $\mathrm{D}$ had significant difference from $C$ with $p$ value 0.005 . There was no significant difference between groups $\mathrm{D}$ and $\mathrm{E}$ with $\mathrm{p}$ values 0.546 .Necrosis was seen in all 10 animals of group A there were 2 animals with mild necrosis and 8 with moderate. For remaining 4 groups necrosis was not observed in any animal. The difference was highly significant with $\mathrm{p}$ value $<$ 0.001. No pair wise comparison was required as necrosis was observed only in aspirin treated group.

Thus it is proved that Group D fluvoxamine in higher dose of 200mg/kg and Group E mirtazapine $60 \mathrm{mg} / \mathrm{kg}$ offers gastroprotection not only by antioxidant potential but also by decreasing inflammatory and ulcerative changes in gastric mucosa induced by aspirin. ${ }^{12}$

\section{Histopathological Analysis of Rats Antral Gastric Tissue: \\ Group A: Aspirin 400mg/kg treated group (positive control)}

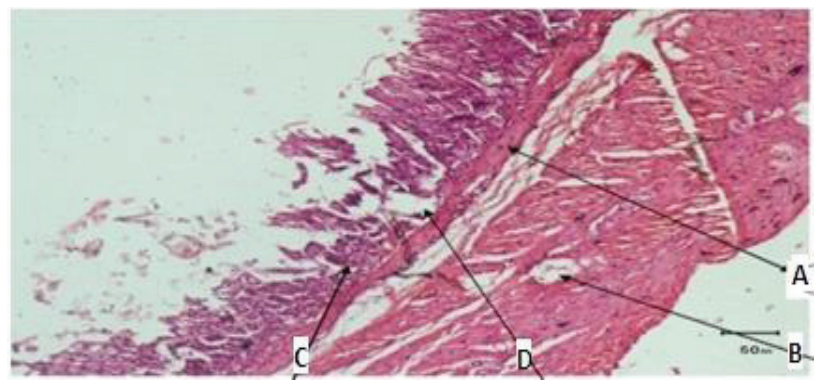

Fig-2: H \&E stained slide of aspirin group showing following changes

A. Severe Cellular infiltration

B. Congestion

C. Severe ulceration involving the entire mucosa

D. Necrosis

\section{Group B: Omeprazole $20 \mathrm{mg} / \mathrm{kg}$ pretreated followed by aspirin $400 \mathrm{mg} 7 \mathrm{~kg}$}

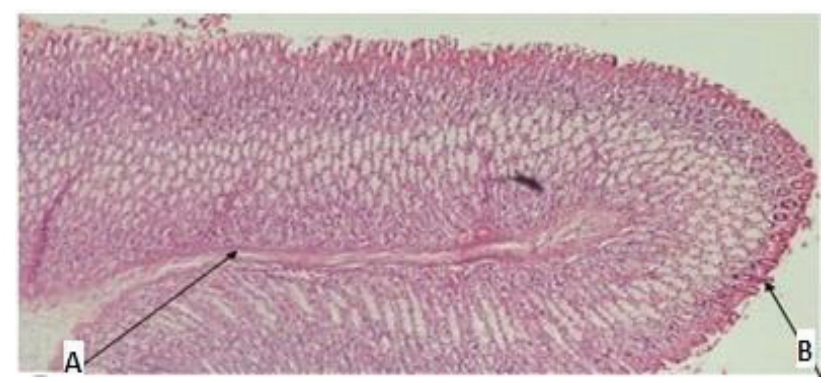

Fig-3: H \&E stained slide of omeprazole group showing following changes:

A. No cellular infiltration and congestion

B. Intact epithelium

Omeprazole pretreatment inhibited the changes like cellular infiltration, congestion and ulceration.

Group C: Fluvoxamine $100 \mathrm{mg} / \mathrm{k}$ pretreated group followed by Aspirin $400 \mathrm{mg} / \mathrm{kg}$

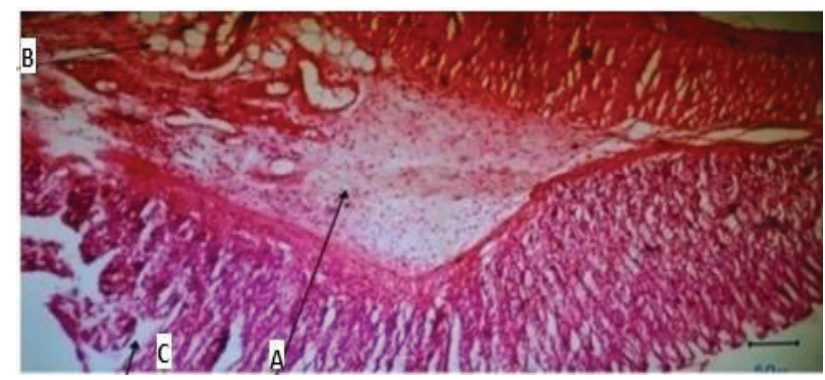

Fig-4: H \& E stained slide of fluvoxamine $100 \mathrm{mg} / \mathrm{kg}$ showing following changes:

A. Mild cellular infiltration

B. Mild congestion

C. Mild ulceration

Group D: Fluvoxamine $200 \mathrm{mg} / \mathrm{kg}$ pretreated group followed by Aspirin 400mg/kg

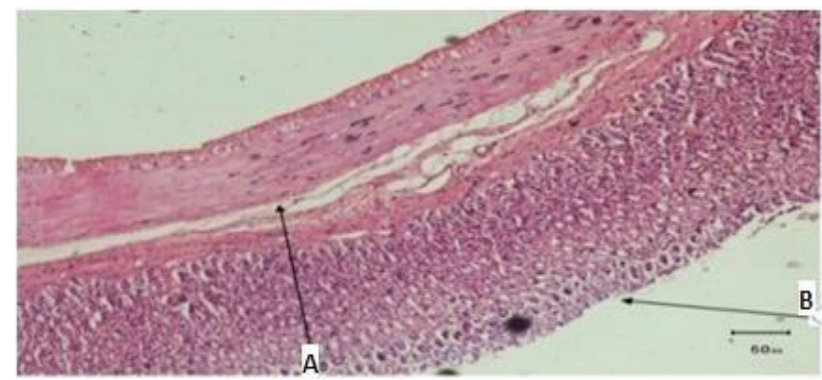

Fig-5: H \&E stained slide of fluvoxamine pretreated group showing following changes.

A. No cellular infiltration

B. Intact epithelium

Fluvoxamine in higher dose of $200 \mathrm{mg} / \mathrm{kg}$ significantly inhibited the Aspirin induced changes like cellular infiltration, congestion and ulceration. 


\section{Group E: Mirtazapine 60mg/kg pretreated group followed by aspirin $400 \mathrm{mg} / \mathrm{kg}$}

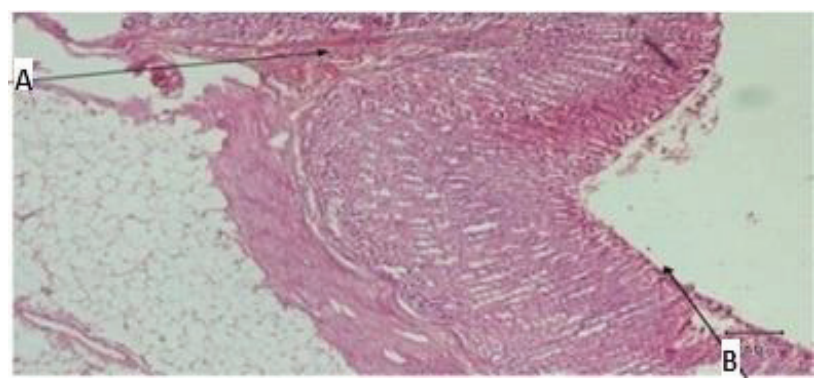

Fig-6: H \& E stained slide of mirtazapine pretreated group showing following changes

A. Mild cellular infiltration

B. Intact epithelium

Mirtazapine in dose of $60 \mathrm{mg} / \mathrm{kg}$ reduced the severity of cellular infiltration and significantly inhibited the changes like congestion and ulceration. No hemorrhage was observed in all.

\section{DISCUSSION}

Depression has been recognized as one of the higher risk factor for causing PUD compounded with NSAID usage. ${ }^{16}$ First line drugs for treatment of mild to moderate depression are Selective serotonin reuptake inhibitors ${ }^{17}$ But they increase the risk of GI bleeding. ${ }^{18}$

Therefore for diagnosed patients of depression there is need for identifying and exploring the drugs possessing both antidepressant and antiulcer activity. The antiulcerogenic effect of fluvoxamine and mirtazapine has been evaluated individually in four studies, two of mirtazapine and 2 of fluvoxamine against indomethacin induced and stress induced ulceration.

Alternatively aspirin was chosen with its wide use and $35 \%$ greater ability to produce ulcers than indomethacin ${ }^{19}$

Secondly gastric hispathological analysis had been done previously only in one study of mirtazapine and one of fluvoxamine in indomethacin and stress induced ulcer. ${ }^{20,21}$

Gastric histopathological evaluation was carried out in the present study and it was observed that gastric damage induced in aspirin treated group was greatly attenuated by prophylactic use of fluvoxamine $100 \mathrm{mg} / \mathrm{kg}$ with mild ulceration, mild cellular infiltration and mild congestion

The effect of fluvoxamine $200 \mathrm{mg} / \mathrm{kg}$ was more pronounced So fluvoxamine $200 \mathrm{mg} / \mathrm{kg}$ completely prevented the aspirin induced ulceration. The definite mechanism by which fluvoxamine suppresses oxidative stress is still unclear however researcher have demonstrated that fluvoxamine interacts with mitochondrial lipid bilayer and affects electron transport chain thereby inhibiting oxidative phosphorylation. ${ }^{22}$ Increase in serotonin level has also reported to be an important mechanism of antioxidant effect in nerves. ${ }^{23}$ Mirtazapine also reduced the effects of aspirin with mild cellular infiltration and no congestion and no ulceration. In a study of mirtazapine, increasing adrenaline levels were shown to have antiulcer activity and this effect was mediated by stimulation of postsynaptic adrenergic receptors. ${ }^{20}$ This could be another mechanism of antiulcer effect of mirtazapine apart from increasing antioxidant level. Finally fluvoxamine in higher dose and mirtazapine displayed significant gastroprotection.

The better efficacy of fluvoxamine $200 \mathrm{mg} / \mathrm{kg}$ in comparison to fluvoxamine in low dose of $100 \mathrm{mg}$ and mirtazapine $60 \mathrm{mg} / \mathrm{kg}$ showed that higher dose of fluvoxamine would have a dual antiulcer in addition to antidepressant in patients suffering from depression. The observed histopathological findings are comparable to work already done on rats using dual doses of fluvoxamine and single dose of mirtazapine $60 \mathrm{mg} / \mathrm{kg}$ by comparing the levels of enzymatic parameters like GSH in gastric tissue ${ }^{12}$

These effects are also comparable to study done on antiulcer activity of mirtazapine, venlafexine and escitalopram in indomethacin induced ulcer, but use of mirtazapine in low dose of $10 \mathrm{mg} / \mathrm{kg}$ offered no significant gastroprotection in above mentioned study. ${ }^{20}$ Thus there is a potential for dual use of antidepressants like fluvoxamine in future with the coexistence of depression and peptic ulcer. Further work is needed to explore the other mechanisms involved in antiulcer effect of these drugs.

\section{CONCLUSION}

Gastric histological analysis revealed significant prophylactic gastroprotective ability of $200 \mathrm{mg} / \mathrm{kg}$ fluvoxamine and lesser in $100 \mathrm{mg} / \mathrm{kg}$ fluvoxamine and $\quad 60 \mathrm{mg} / \mathrm{kgmirtazapine}$ versus $20 \mathrm{mg} / \mathrm{kg}$ omeprazole in aspirin induced damage.

\section{REFERENCES}

1. Bartolumucci A, Leopardi R. Stress and depression preclinical research and clinical implications. PLoS One .2009. 4.

2. Hsu CC, Hsu CY, Chang KH, Lee CY, Chong LW, Lin CL. Depression and risk of peptic ulcer disease. Medicine.2015; 94; 1-8.

3. J Ainu M, Device SS. Antiulcerogenic and ulcer healing effects of Solanum nigram on 
experimental ulcer models. $\mathrm{J}$ of Ethno Pharmacol. 2006; 104: 156-63.

4. Andreoli T, Chan PD, Cowell JC, Gilbert DM, Green C, Johnson, M. et.al. Management of patients with Gastric and duodenal disorders in Brunner and Suddarth's. Testbook of Medical Nursery. $11^{\text {th }}$ edition 2008; 1203-1279.

5. Kang JY, Elders A, Mejeed A. Recent brands in hospital admission and mortality rates for peptic ulcer. Ailment pharmacol thr. 2006; 24: 65-79.

6. Borro Sk, Log Isety Rk, Makela GR Antiulcer effect of Alovera on NSAIDS indvcel ulcer in rats. Afr J phaun pharmacal. 2011; 5: 1867.

7. Oshima T. Aspirin induces gartic epithelial barrier dysfunction by activating p38 MAPK Vial Claudin 7. Am J Physiol. 2008; 3:800-806.

8. Gery B, specheler SJ, Peptic Ulcer disease GIT and live disease. 8th edition Philadelphia 2006; 1089-1110.

9. Roy C, Das SK .Potential role of Aegle marmales in aspirin induced ulcer. Saudi J. Gastroenterol. 2012; 3: 188-194.

10. Awaad AS, El-Meligy R, Solman G.D. Natural products in Treatment of ulcerative colitis and peptic ulcer. Journal of Saudi Chemical society. 2012; 17: 101-124.

11. Kinoshita $\mathrm{Y}$, Ishimura N, Ishihara $\mathrm{N}$. Advantages and disadvantages of long term proton pump inhibitorsuse. J Neurogastroenterol motil. 2018; 24: 182-196.

12. Fatima S, Bhatti M, Alam SS. Antiulcer effect of fluvoxamine, mirtazapine and omeprazole in aspirin induced ulcer in rats. Proceedings S.Z.P.G.M.I. 2017; 31: 62-70.

13. Kocer B, Surmeli S, Solak C, unal B, Bozkurt B, Yildirim $\mathrm{O}$ et.al. Factors affecting mortality and morbidity, in patients with peptic ulcer perforation. J. Gastroenterol. 2007; 4: 565-570.

14. Dursan H, Bilici M, Albayrak F, Ozturk C, Saglam MB, Alp HH. et.al. Antiulcer activity of fluxamine in rats and its effect on oxidant and antioxidant parameters. BMC Gastroentrol. 2009; 9: 9-36.

15. Billici M, Ozturk C, Dursun H, Albayrak F, Saglam MB, Uganik A, Gulaboglo M, takin SB. Protective effect of mirtazapine on indomethacin induced ulcer in rats and its relationship with oxidant and antioxidant parameters. Dig Dis Sci. 2009; 54: 1968-75.

16. Choas C, Choas Yi, Chang K, Lee YC, Chong LW, Lin LC, Sung CF. et.al. Depression and risk of peptic ulcer disease. Medicine (Baltimore). 2015; 94(51).

17. Susan C. Sharp, Jessica A. Helling. Efficacy and Safety of Selective Serotonin Reuptake Inhibitors in the Treatment of Depression in Children and Adolescents. Clin Drug Invest. 2006; 5:247-255.

18. LokeY, Trevidi AN .Gastrointestinal bleeding due to SSRI and NSAID. Ailment pharmacol ther. 2011; 1:31-40.

19. Heinekens $\mathrm{CH}$.Benefits and risk of aspirin for primary and secondary prevention of cardiovascular diseases. 2014; 12.147-154

20. Alawdan SA, Zaki HF. gastroprotective activity of mirtazapine, esctilaopram and venlafexine in depressed rats. African journal of pharmacy and pharmacology. 2013; 7: 2701-9.

21. AM Elsaed WM, Alamadhe AM, Ahmadi BT, Taha JA, Tara bishi RM. Gastroprotective and antioxidant effect of fluvoxamine in stress induced ulcer in rats. 2018; 13(5): 422-431.

22. Zafir A, Ara A, Banu N. Invivo antioxidant status A putative target of antidepressant action. Prog Neuropsychopharmacol Biol psychiatry. 2009; 33(2): 220-228.

23. Heuther Schuff WP. platlet serotonin act as locally releasable antioxidant. Adv Exp Med Biol. 1996; 398: 299-306.

\section{The Authors:}

Dr. Shahnaz Fatima

Assistant Professor,

Department of Pharmacology,

Sahara Medical College, Narowal.

Dr. Maira Bhatti

Assistant Professor,

Department of Pharmacology,

Sharif Medical College, Lahore.

Prof. Saadia Shahzad Alam

Head, Department of Pharmacology,

Shaikh Zayed Medical Complex, Lahore

\section{Corresponding Author:}

Dr. Shahnaz Fatima

Assistant Professor,

Department of Pharmacology,

Sahara Medical College, Narowal.

E-mail: drshahnazfatima82@gmail.com 